\title{
Téoros
}

Revue de recherche en tourisme

\section{L'état de la recherche en tourisme au Québec} Les grandes lignes d'un colloque

\section{Hélène Dostie et Lynda Johnson}

Volume 1, numéro 2, 2e trimestre 1982

La recherche en tourisme : forces et faiblesses

URI : https://id.erudit.org/iderudit/1080852ar

DOI : https://doi.org/10.7202/1080852ar

Aller au sommaire du numéro

Éditeur(s)

Université du Québec à Montréal

ISSN

0712-8657 (imprimé)

1923-2705 (numérique)

Découvrir la revue

Citer cet article

Dostie, H. \& Johnson, L. (1982). L'état de la recherche en tourisme au Québec : les grandes lignes d'un colloque. Téoros, 1(2), 2-4.

https://doi.org/10.7202/1080852ar d'utilisation que vous pouvez consulter en ligne.

https://apropos.erudit.org/fr/usagers/politique-dutilisation/ 


\title{
L'état de la recherche en tourisme

\author{
Les grandes lignes d'un colloque \\ Par Hélène Dostie et Lynda Johnson
}

A l'heure oủ les loisirs, les vacances et le tourisme font l'objet par pans entiers de bouleversements majeurs, très paradoxalement une question reste encore posée: quel est l'état de la recherche en ces domaines au Québec? Un récent colloque sur la recherche en tourisme interdit de proposer encore une fois une réponse assurée.

En l'absence d'une véritable politique québécoise du tourisme, des intervenants de différents secteurs du tourisme ont été appelés, le 3 mars dernier à réfléchir ensemble sur la question de la recherche: tâche délicate. les définitions et les concepts étant variés. S'il est vrai que de temps à autre, des concensus se forment... à tout instant de nouveaux cas douteux apparaissent.

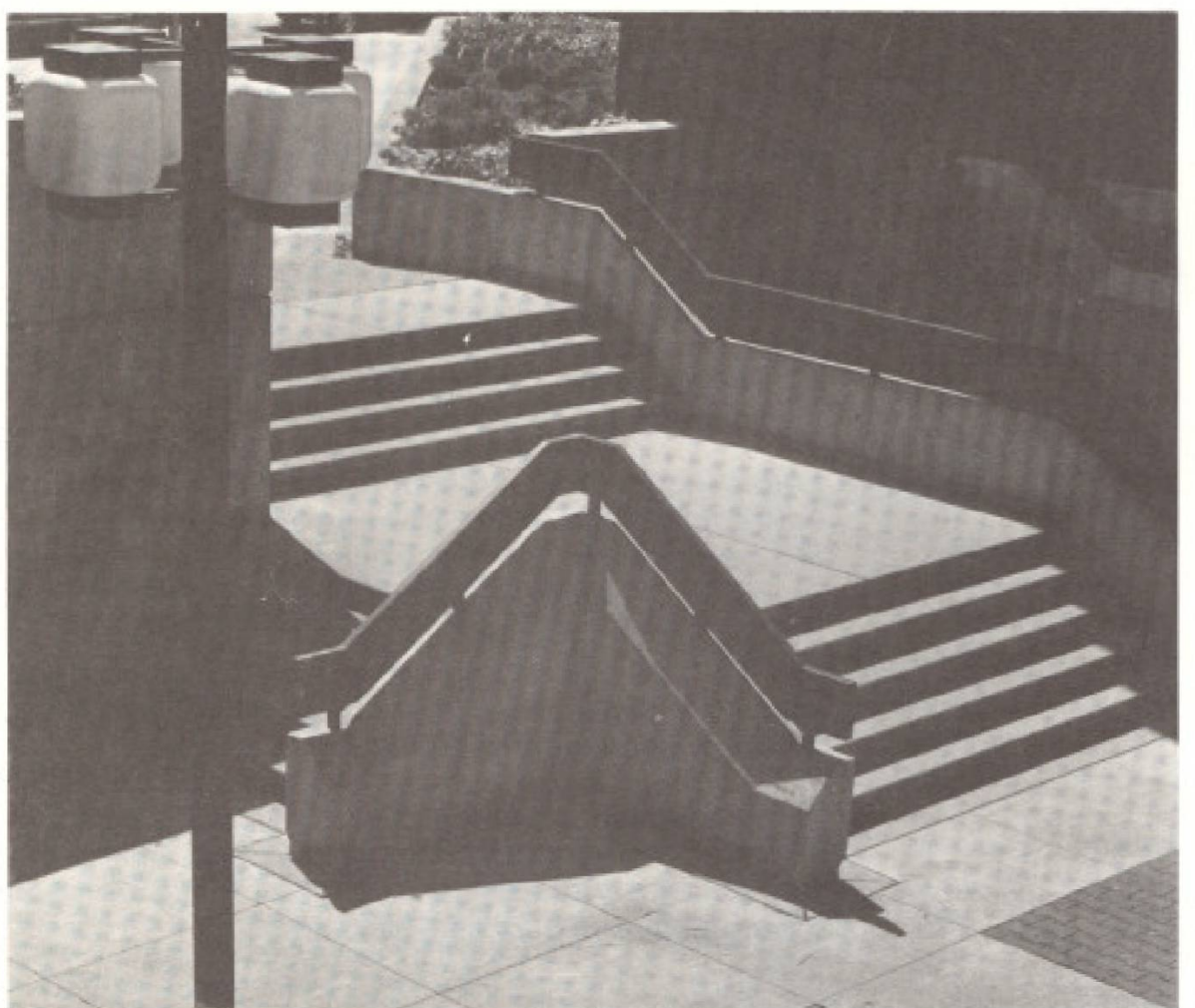

\section{Qu'est-ce que la recherche?}

On entend déclarer ces temps-ci avec force que la recherche en tourisme n'existe pas. Mais on dit aussi qu'elle existe. Comment peut-elle exister et ne pas exister en même temps? Précisément, quelle est-elle?

Selon le Petit Robert" "la recherche est /a quête d'informations nouvelles nous aidant à la découverte de connaissances et de lois nouvelles pour étudier une question." Cette définition paraît simple, peut-être, mais les qualificatifs qui sont attribués à la recherche la complexifient davantage. Ne parle-t-on pas de recherche fondamentale, de recherche appliquée, de rechercheaction? Ainsi, le secteur universitaire semble se spécialiser dans la recherche fondamentale tandis que le secteur privé conçoit des recherches appliquées et le milieu associatif oriente souvent sa recherche vers l'action concrète. Pour plusieurs, il faut savoir distinguer entre le concept de recherche et celui d'étude. D'autres ne jugent pas pertinente cette distinction.

II appert toutefois que ciest à travers une analyse et une réflexion rigoureuse de la recherche dans son ensemble que I'on arriverait à solutionner les problèmes soulevés à travers le temps et l'espace de la recherche au Québec. Les quoi, qui, comment, pourquoi doivent ètre posés afin de rendre l'action plus süre et atteindre les objectifs posés au départ par les chercheurs.

Historiquement, ces questions d'ordre épistémologique ont été maintes fois soulevées, laissant finalement apparaître l'urgence d'une recherche structurée afin d'assurer un développement touristique sain. Déjă Albert Tessier dans son rapport sur le tourisme en 1939, démontrait que la recherche était nécessaire. De par une "action méthodique, tenace et coordonnée", le produit conçu serait original et spécifique à la culture québécoise. Nonobstant cette prise de conscience, on n'offrirait aux touristes qu'une imitation à I" américaine" (1). Par contre, une recherche structurée et orientée vers l'avenir amènerait un developpement plus solide et avantgardiste.

Un colloque sur la recherche en tourisme au Québec

C'est dans cet esprit que le Centre d'études du tourisme (CET) et le module de Gestion et intervention touristiques (GIT) de I'Université du Québec à Montréal organisaient un premier colloque sur la recherche en tourisme au Québec. Les objectifs qu'ils s'étaient proposés, fort ambitieux d'ailleurs, visaient à dresser un bilan critique de la situation actuelle. On souhaitait, ainsi, dégager les lignes de force et les priorités futures de la recherche.

A cet effet, Monsieur Jean Stafford, professeur au module GIT et quelques étudiants avaient élaboré un texte dans l'espoir de susciter des interrogations 
en rapport avec la recherche au sens global du terme. On tentait d'y préciser une démarche de travail apte à générer une action plus adéquate et plus structurée. Pour ce faire, selon eux, il convient d'identifier les lacunes. l'ampleur et les impacts de la recherche. On doit, par la suite, évaluer les coûts globaux en rapport avec la rentabilité désirée. La méthodologie choisie selon une problématique arrêtée doit être précédée diune réflexion sur l'utilisation de la recherche pour l'organisme. Dans ce cadre, les indicateurs retenus facilitent le constat des tendances actuelles et laissent transparailtre les changements sociétaux survenus. Ils apportent, par conséquent une rigueur décisionnelle. orientée vers l'action qui tient compte des besoins identifiés. Quoique constituant en soi une excellente source de référence, la densité de ce texte limitera toutefois son usage lors du colloque.

Au niveau de la participation, les organisateurs du colloque souhaitaient une répartition équitable entre les différents milieux d'intervention: universitaire, privé, associatif et gouvernemental. Dans les faits, on remarqua, par contre l'absence quasi totale des Associations touristiques régionales (exception faite de I'A.T.R. du Bas St-Laurent) et des firmes privées. Cette absence affecta l'écquilibre des échanges, déjà compromis par le grand nombre d'utilisateurs de recherches par rapport aux concepteurs.

Mais le colloque aura toutefois permis de prendre connaissance d'une toute nouvelle recherche entreprise par Marcel Samson, chercheur à I'Institut National de Recherche Scientifique Urbanisation et Véronique Barnier de l'Université de Montréal. Un sujet toujours d'actualité: "Tes vacances des Montréalais". Depuis 10 ans, le pourcentage des Montréalais qui prennent des vacances n'a pas augmenté. Conclusion désarmante qui nous amène à s'écrier: oui, il faut de la recherche sur le developpement futur du tourisme mais aussi sur les motivations, les besoins et les habitudes de voyages.

Les visions particulières... ou les premiers coups de sonde.

Plusieurs personnes insistent sur la corrélation étroite qui existe entre la recherche en tourisme et le développement de cette forme de loisir. Pourtant, quoiqu'elles s'entendent globalement sur le principe, elles ont une vision fort différente de la recherche. Ces perceptions nous ant d'ailleurs été présentées inévitablement au panel d'ouverture.
Le colloque s'est déroulé en deux parties distinctes. La première permit à des conférenciers des différents secteurs d'exprimer leur vision particulière de la recherche, d'effectuer leur premier coup de sonde, et la seconde favorisa les libres échanges dans le cadre d'ateliers et d'une plénière-synthèse. Le ministère de I'Industrie, du commerce et du Tourisme, invité a participer à ce panel, s'excusa au tout dernier instant. Faut-il croire qu'il était occupé à régler des tâches plus urgentes? Nous ne nous permettrions pas de l'affirmer avec certitude. Pour Roger Nadeau, du département de géographie de I'Université de Sherbrooke, l'état présent de la recherche est directement lié aux efforts consentis. Et comme les. corps publics s'attardent à des domaines quils jugent plus pertinents, les chercheurs en tourisme se voient isolés, sans aucun moven technique de réalisation et de diffusion. En l'absence d'orientations gouvernementales claires, il est urgent, dit-il, que le secteur universitaire se dote d'objectifs communs afin d'aider au développement du tourisme.

"Dans les pays, où il se fait le moindrement de recherches organisées en tourisme. IUniversité joue un róle de pointe, un róle de chef de file en ce domaine. Mais, au Québec, il n'y a ni chef, ni file..." (2)

Claude Lamothe, de la firme Sotar. aborda la question d'une manière quelque peu différente. II traça un bilan de la recherche de la Révolution tranquille des années 60 à nos jours. II dégagea dans ce retour historique des priorités de recherche pour que le Québec cesse d'être: "a la remorque des autres pays dans le domaine de la recherche en tourisme" (3). II est temps que le Québec cesse d'imiter à gauche et a droite et commence dès maintenant à innover dans le domaine touristique. Pour ce faire, toute une gamme de recherches devraient etre entreprises permettant une meilleure structuration gouvernementale, et des innovations au niveau de l'accueil, de I'hébergement et des autres secteurs connexes à l'industrie touristique.

Bref, il est urgent pour le Québec de se donner des outils pour une meilleure planification du développement touristique et qu'il mette de côté le hasard comme élément de solution.

II faut toutefois considérer que le secteur prive a des objectifs quelque peu différents. La priorité n'est pas d'effectuer des recherches générales mais de répondre aux besoins des clients. Pour certaines entreprises, comme par exemple, les multinationales, les recherches consistent principalement en des études de marché, de clientèle, d'analyse coûtsbénéfices. Ces recherches, pour la plupart liées à leurs stratégies de développement, sont confidentielles.

Le dernier conférencier, Louis Jolin, professeur en tourisme à I'UOAM et président du secteur Tourisme du Regroupement des organismes nationaux de loisir du Québec, se fit l'habile deffenseur du milieu associatif lié au tourisme social en soulevant la nécessaire corrélation entre les objectifs et actions prônés par les associations et le développement touristique futur du Québec. II faut blâmer, dit-il la structure administrative québécoise, malgré le beau discours tenu au Sommet touristique de Sherbrooke (1978) et l'existence du Conseil du Tourisme. Il importe de créer un lieu commun de concertation pour mieux dégager les priorités futures de la recherche et de constituer un fonds de recherche spécifique en tourisme qui pourrait ètre géré par ce lieu de concertation. II relève comme significatif |'enthousiasme du milieu associatif, un exemple que devraient suivre les autres secteurs d'intervention.

"Plusieurs études ont eté produites
par les associations de tourisme
social qui traduisent une relative
vitalite... leur principal merite est de
de ne pas etre trop corporatistes... un
autre caractere est d'etre engagées.
voire militantes... elles ne sont pas
neutres comme toute recherche
d'ailleurs, mais au moins, elles ne le
laissent pas croire..." (4)

La fouille systématique

Dans le cadre des ateliers qui suivirent. les intervenants ont défini la problématique autour de certains axes majeurs: I'élaboration des données, la diffusion de la recherche, la concertation et plus encore, la perception même du tourisme en tant que science.

L'émergence récente de la réalité du voyage explique, en partie, le peu d'importance et de réflexion qu'on lui a accordé jusqu'à présent. On a souvent considéré le tourisme comme un fait acquis sans nécessité d'organisation. un phénomène marginal et impalpable. D'oủ l'inexistence d'une politique globale qui accorderait autant d'importance au social qu'à l'économique. Or jusqu'à présent, on s'est attardé beaucoup trop aux seuls aspects économiques en soulignant notamment la non-rentabilité du tourisme social sans reconnaître ses impacts réels. 
Mais, doit-on attendre des corps publics qu'ils fixent les orientations générales pour tous, ou si par contre, à la base, dans les divers milieux, ne devrait-on pas les fixer et les ordonner selon des intérêts spécifiques? La recherche estelle vouée à l'échec s'il y a absence de politique de développement touristique? Autant d'interrogations fondamentales qui laissent apparaître un malaise certain. Et s'il y a politique, devrait-elle être sectorielle ou globale? Question pertinente et importante, mais difficile à répondre. Une chose qui semble évidente toutefois, est qu'une politique sectorielle ne facilitera pas une concertation qui tarde à venir. Comme le disait Roger Nadeau, les chercheurs sont déjà si isolés, dans un univers où la compétition et la spécificité des intérêts génèrent luttes et conflits, sans qu'il soit utile de paralyser plus longtemps I'avancement de la recherche. En outre, I'absence de chercheurs ou leur manque de spécialisation, peut-être, entraînent un manque de leadership. Mais encore ici, la question est de savoir qui serait en mesure de mieux assumer ce rôle et de revendiquer par exemple, un fonds de recherche si nécessaire? Nul doute qu'un tel fonds puisse solutionner en partie le manque de diffusion des écrits noté lors du colloque.

Pourtant, la problématique de la recherche ne repose pas seulement sur une non-disponibilité des données, elle est aussi causée par une déficience de l'information. Les données sont bien souvent partielles, périmées, voir mème empruntées. II s'ensuit donc une incohérence et un manque de scientificité des informations accessibles. En outre, comme il n'existe aucun concensus au niveau des concepts, les recherches se font sur des bases différentes et sont ainsi difficilement comparables.

\section{Des avenues possibles}

Toutefois, l'état de la recherche n'est pas aussi précaire qu'il le semble à priori. Au colloque, les participants ont soulevé des avenues positives qui, si elles étaient embrassées, règleraient les déficiences notées précédemment.

Avant tout, il importe de sientendre sur la définition des concepts et de se doter d'objectifs communs et d'une méthodologie commune. Ce premier pas engendrerait une collaboration entre les secteurs, revitalisant ainsi la recherche et communiquant aux médias, actuellement désintéressés de cette question, un enthousiasme nouveau et non négligeable.

La modernisation et l'informatisation des outils de recherche sont d'autres moyens pertinents permettant d'articuler une réflexion positive pour le développement du tourisme. Devant I'ampleur bibliographique. I'on devra bientôt assurer une télé-informatisation des données, un catalogage et une modernisation des instruments. Et puis. la formation adéquate de gestionnaires de recherche dans les institutions d'enseignement et dans les entreprises amènerait une confiance et une compréhension des instruments utiles à la recherche.

Enfin, et c'est peut-être le plus fondamental, n'y aurait-il pas lieu d'encourager des initiatives de création de centres d'intére̊ts pour la recherche touristique quitte à privilégier une concentration plus efficace à un éparpillement trop démocratique. De plus, un lieu commun de concertation aurait pour effet de dégager des priorités de recherche et d'accroitre la diffusion des recherches en cours. Un fonds de recherche en tourisme pourrait être un important stimulant.

Néanmoins, on peut se demander si le problème de confidentialité sera ainsi résolu. Chacun semble vouloir rester sur sa position défendant ses intérêts spécifiques. Cependant. jusqu'à présent, nous n'avons jamais remarqué que la collaboration vouait à l'échec. En général, tous y donnent mais tous y gagnent aussi. On peut prendre l'exemple des organismes du secteur associatif qui, unis dans leurs efforts. arrivent à une action plus cohérente et plus efficace.

Mais toutes ces recommandations du 3 mars dernier sont loin d'être nouvelles, on les avait déjá proposées dans une certaine mesure chez Sotar, lors de la création du CET à la Chambre de Commerce du Québec..

Certains répondront que la concertation va à l'encontre de la liberté des chercheurs. Mais "les ressources sontelles si infinies que les chercheurs euxmêmes soient dispensés de s'en soucier au nom de quelques privileges sur tous les autres groupes de la societé et fréquemment aux dépens des études urgentes," (5) Serait-ce de contrer la liberté que de permettre une meilleure utilisation des recherches par le milieu? Sur ce, nous croyons que les chercheurs pourraient trouver, dans le cadre d'une concertation, des intérêts communs tous en conservant leur liberté d'action. En autant qu'ils répondent aux besoins des utilisateurs pour qui la recherche est nécessaire.

Nonobstant certaines lacunes, il nous apparaît que, globalement, la tenue de ce colloque a été un geste positif. Cette rencontre fut un moment de prise de conscience collective sur les besoins impérieux de faire de la recherche et d'en assurer une continuité. En outre, il aura permis de se familiariser avec des projets enthousiastes qui s'amorcent. Déjă l'on commence à sentir de la vitalité dans certains milieux. qui n'attendent pas une politique de recherche. Voici quelques exemples parmi les plus récents:

Le Centre d'études du tourisme fait peau neuve: depuis septembre dernier, on se réorganise. On classe des documents, on met à jour les fichiers, on complète les dossiers. Bref, tout pour ëtre en mesure d'ouvrir à tous l'information.

Teoros s"implante dans le milieu: un nouvel outil que se donne le module GIT qui comblera espère-t-on une carence parmi les écrits actuellement disponibles. Les cahiers de recherche Teoros publieront des rapports de recherche, des études scientifiques sur I'une ou l'autre question reliée au phénomène touristique. IIs veulent susciter une collaboration entre les chercheurs universitaires ou autres, en les invitant à s"associer selon leur intérêt propre aux différents thèmes abordés.

Le Groupe de ressources en tourisme social se forme: expérience positive qui démontre le souci des associations gestionnaires de tourisme social de réaliser des actions concrètes dans le cadre d'intérèts et d'objectifs partagés conjointement avec des regroupements d'usagers. II a I'avantage d'insuffler au milieu associatif un nouveau dynamisme.

Ces nouveaux projets ouvrent la porte un esprit de coopération. Certains pourront rétorquer que cet activisme ne solutionnera pas tous les problèmes, mais il demeure que des projets seront de puissants générateurs pour l'avenir.

"Ceux qui songent aujourd"hui, au sort de la recherche devraient s'en souvenir..

IIn'y a pas de science sans espérance et il n'y a pas de méthode sans désir. (6)

Notes

(1) TESSIER, Albert. Rapport sur le tourisme. 1939

(2) NADEAU, Roger. Extrait de la conference donnese le 3 mars 82

(3) LAMOTHE, Claude. Extrait de la conference donnte le 3 mars 82.

(4) JOLIN, Louis. Extrait de la conférence donnée le 3 mars

(5) GAGNE, Jean. Liberte et planification de is recherche, Le Devoir, 12 mars 1902

(6) DUMONT. Fernand. Le sert de ta cuhtere Le Devoir, 12 mars 1982 